\title{
ISO 15531 MANDATE: A Product-process-resource based Approach for Managing Modularity in Production Management
}

\author{
A. F. Cutting-Decelle, ${ }^{1}{ }^{*}$ R. I. M. Young, ${ }^{2}$ J. J. Michel, ${ }^{3}$ R. Grangel, ${ }^{4}$ J. Le Cardinal ${ }^{1}$ and J. P. Bourey ${ }^{5}$ \\ ${ }^{1}$ Industrial Engineering Research Laboratory, Ecole Centrale Paris, Chatenay Malabry, France \\ ${ }^{2}$ Department of Mechanical and Manufacturing Engineering, Loughborough University, Loughborough, UK \\ ${ }^{3}$ IDPICONSEIL, Maisons-Alfort, France \\ ${ }^{4}$ Departament de Llenguatges i Sistemes Informàtics, Universitat Jaume I, Castello, Spain \\ ${ }^{5}$ Industrial Engineering Team, Ecole Centrale Lille, Villeneuve d'Ascq, France
}

\begin{abstract}
Managing modularity and commonality in product development more and more needs modularity and commonality in the production process, with the objectives of reducing manufacturing costs, time to market and improving quality. A critical issue is the way of managing data, information and knowledge: data most of the time structured according to data models, often using proprietary formats, leading to consistency problems for the exchanges. The use of international standards is a good way of improving quality of the information systems used in production management, since they facilitate interoperability of the software tools used. They also contribute to the integration of the production process in a product life cycle management-based approach. This study presents the ISO 15531 MANDATE standard for the exchanges of industrial manufacturing management data. In terms of industrial maturity, MANDATE is a new standard, whose development is based on research work done by the authors and whose parts have not reached the IS status (necessary for sake of stability) at the same time. For this reason, the different models proposed by the standard have not been implemented altogether at the same time. Indeed numerous standards do exist in the domain of production information management, however the information models proposed are not always compatible in between them, the vocabulary used is not defined in the same way even though the terms used are the same: ontology-based approaches are sometimes necessary to find the common 'essence' of the information handled, but they can be integrated in software interfaces, thus making easier to convey a higher level of semantics in the exchanges. This study presents one of those approaches, defined in the INTEROP NoE EC funded project.
\end{abstract}

Key Words: manufacturing management, ontologies, information models, modules, international standard, knowledge management.

\section{Introduction}

Production systems (PS) are most of the time large systems where primary inputs are production requirements, production concepts, systems parameters, raw materials, and components and other production management features, and primary outputs are final products, quality of those products, and whatever kind of product related information. From this point of view, their performance can generally be analyzed through five points of view: planning, scheduling, simulation, control, and execution. Those steps are fundamental for the management of production systems.

Planning process represents the periodical activity and is aimed at obtaining the best scheduling of material flow. Planning in manufacturing can be difficult. Planning has to deal with detailed data, summary

*Author to whom correspondence should be addressed.

E-mail: afcd@skynet.be

Figures 2 and 5-7 appear in color online: http://cer.sagepub.com data, internal-external data, subjective information, and sometimes with no information at all.

Planning and scheduling in PS may be defined as the process of allocation of limited resources to production tasks on the basis of such information as for example: machine characteristics, production requirements, time of performance, production constraints, and economical factors. The control system determines through the use of control technologies the sequences of control action for the resources used in the actual manufacturing process.

One of the roles of execution is to follow the performance of the system and to give backward information for the control system, which on the basis of this information creates the new available sequences of control action. The phases: planning, scheduling, control, and execution may be more concurrent and it is necessary to take into consideration the cycle time for each of the phases and also for the whole cycle. The common approach to the problems was based on a rigid, sequential, one-way set of deliverables leading eventually to a finished system. Usually by the time the 
system was finished, the requirements had changed. Newer systems development methodologies are based on rapid, concurrent prototyping with frequent feedback to validate the requirements. For Frankovic et al. [1], realization of such approach requires parallel coordination, rapid obtaining and treating the information, and also rapid communication.

The concept of modularization has attracted an increasing attention in the last few years. The meanings and purposes of modularization in industry vary between regions and companies [2]. There is no clearcut definition of the term shared by the whole industry. Yet, there does exist a feature relatively common across various practices of modularization in the industry. It entails having larger units in subassembly and also often involves outsourcing these subassemblies to suppliers (as most frequently observed in the European auto industry). This fact suggests that there are at least three facets in the phenomenon called 'modularization': (1) 'modularization in product architecture' (modularization in design) which has been discussed quite often in the field of the management of technology; (2) 'modularization in production;' and (3) 'modularization in inter-firm system' (outsourcing subsystems in larger units to outside suppliers). These three facets have often been mixed up, causing confusion in discussing modularization.

Managing modularity and commonality in product development more and more needs modularity and commonality in the production process, with the main objectives of reducing manufacturing costs, improving quality of the products, and reducing time to market. A critical issue in managing modularity is the way of managing all the data, information, and knowledge circulating during the production process: those data are most of the time structured according to given data models, most the time using proprietary formats. Further, this structure is not consistent throughout the production process, the semantics embedded is often poor and the exchanges among the tools made neither straightforward nor easy to automate. The approaches to information exchanges are often syntactic rather than semantic-based, whence problems in knowledge sharing and re-use - and important problems in terms of interoperability of the software tools used.

The use of international standards is a good way of improving quality of the information systems used in production management, since they facilitate interoperability of the software tools used, also because they contribute to the integration of the production process in a product life cycle management-based (PLM) approach. However, the information models commonly proposed in the literature are rarely compatible, the vocabulary used is not defined in the same way even though the terms used are the same: ontology-based approaches are sometimes necessary to find the common 'essence' of the information handled, they also provide powerful interfacing tools since they can be integrated in software interfaces, thus making easier to convey a higher level of semantics in the exchanges. This study presents one of those approaches, defined in the INTEROP EC funded project.

This study proposes a way of managing modularity in production management systems through the use of standardized information models. In the first section, a modular approach to production management is proposed; this modularity comes here from the separate processing of the concepts of 'product', 'process', and 'resources'. This modularity is a fundamental aspect of the ISO 15531 MANDATE standard, in the domain of manufacturing management, presented in the next sections. Industrial applications of the standard are then suggested, on the basis of previous work providing a partial validation of the standard. This standard defines a new emerging paradigm in terms of manufacturing management information, taken as a whole and integrated as such through a systemic approach considering at the same time product, process, and resource concepts. Since the standard has recently reached the IS (International Standard) level, few applications have been developed till now, and only at a partial implementation level.

The objective of this study is to present the theoretical approaches, based on research work developed by the authors of this study, underpinning the principles followed in the development of the standard. Another objective is to present the contribution the standard brings in production management.

\section{A modular approach to production management}

This section deals with some fundamental issues in the way of managing manufacturing related information and knowledge. This information is grouped into three categories, product-, process- and resource-related information, thus defining a basis of the modules developed in the standard. The diversity in the definitions of those concepts is presented in a synthesis table. A modular approach to production management is then proposed. A product-process-resource representation, issued from an ontology-based analysis is also presented in this section.

\subsection{Terminology issues in manufacturing management information}

Manufacturing businesses are becoming more and more globally disseminated and companies are becoming more willing to work closely together in order to remain competitive. It is therefore essential that information and knowledge sharing systems are able to 
support the global nature of business interactions, as mentioned in [3]. product life cycle management (PLM) and enterprise resource planning (ERP) systems offer effective support for communication, but for substantial additional benefit to be gained, there is a need to share information at a level where computational sharing is possible. The resolution of this issue requires an improved definition of the information and knowledge thus enabling a better computational sharing.

The range of information to be shared is not only wide, but needs to be viewed from different, multiple, perspectives. This is because each team member is likely to be interested in different aspects of the information, such that the significant attributes involved will be different and have different levels of significance. Similarly, the need for a location fit in a mechanical product has implications for the assembly dimensions and tolerances, which in turn will have implications for the manufacture of the components required. In addition to viewpoint dependency, there is also a time dependency on information. The existence, and relevance, of particular information will vary with time through the design process.

Perhaps one of the best known efforts to provide common models defining a basis for data exchange and sharing has been done through the work of the ISO in the ISO TC $184 / \mathrm{SC} 4$ committee. This has been in progress for over two decades now, has made some significant progress, but still has much work to do. It is interesting to note that the early work focused on geometry sharing, an area where the meaning of the terminology is shared and clearly understood by the experts involved. However, as one progresses towards areas of information where the terminology is less rigorously defined in product design and manufacture one starts to face problems related to the definition of the terms used. Either common terms are used to mean different things or different terms are used to mean the same thing which leads to potentially substantial interoperability problems, see [4]. The set of terms listed in Figure 1, see [5] illustrates a sub-set of definitions drawn from international standards which provide definitions for 'process', 'product' and 'resource'. It is interesting to note that when comparing these definitions, it is not easy to find out commonalities among the terms!

This problem highlights the need for precise definitions of the terms used - this will clearly appear in the content of the different information models presented in this study.

\subsection{Need for a modular approach}

While PLM offers a range of tools to support the business including the ability to manage workflows, the heart of an effective PLM system is the database at its core. The issue then is how to structure the databases at the heart of PLM in order to ensure all users have access to effective information support. The importance of product models has long been recognized in providing a core of product information to support decisions as mentioned in [6]. However, given the design, manufacture, operation, and disposal aspects of the life cycle, it is also important to support decisions with non product specific information focused on each of these areas of the life cycle. For example, the manufacturing area of the life cycle should be able to offer support on manufacturing process capability and information on suppliers with resources capable of meeting specific capability requirements. This leads to the concept of a product model at the heart of a data/ knowledge based environment, with further bases of data and knowledge to support each of the life cycle phases.

This top level framework for information and knowledge can then be used as a basis to develop formal information and knowledge classifications for each of the life cycle phases, see [7]. Figure 2 illustrates the framework at the heart of a product life cycle representation. It highlights in particular two unified modeling language (UML) class structures which start to provide contexts for manufacturing knowledge sharing:

- The first is for manufacturing capability models which can be used to build representation of an enterprise's manufacturing ability;

- The second is a product model representation which goes beyond typical representations of product characteristics, such as geometry and product architecture, to include other key class relating to product purpose and views which enable life cycle contexts to be captured.

Those two class structures can be seen as modules of the production system, one dealing with information about manufacturing and resources capabilities, the other related to product information.

Modularization has been the subject of many research studies, particularly in the domain of conceptual frameworks, a mandatory stage towards the re-structuring of the enterprises engaged in this process. Among those studies, Takeishi and Fujimoto [2] analyze practices of modularization implemented in the automotive industry. Their purpose is to discuss the concepts of 'modularization in product system,' 'modularization in production system,' and 'modularization in inter-firm system' within the same framework, and to identify the differences and linkages between them. This framework is based on the concept of 'multiple hierarchies.' It sees development-production activities for automobiles as multiple, interlinked hierarchies. It contends that the 


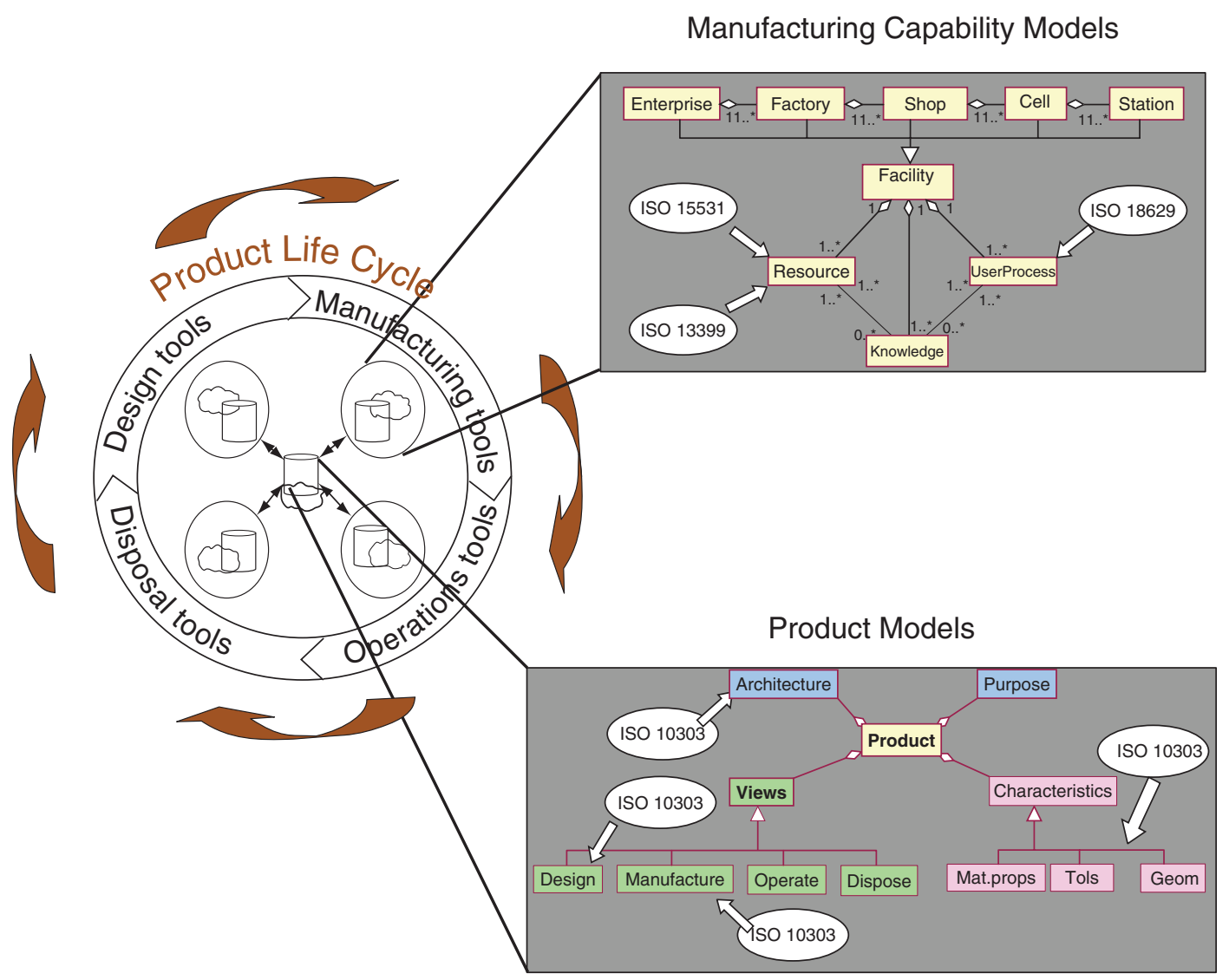

Figure 2. Product information and manufacturing contexts in the life cycle.

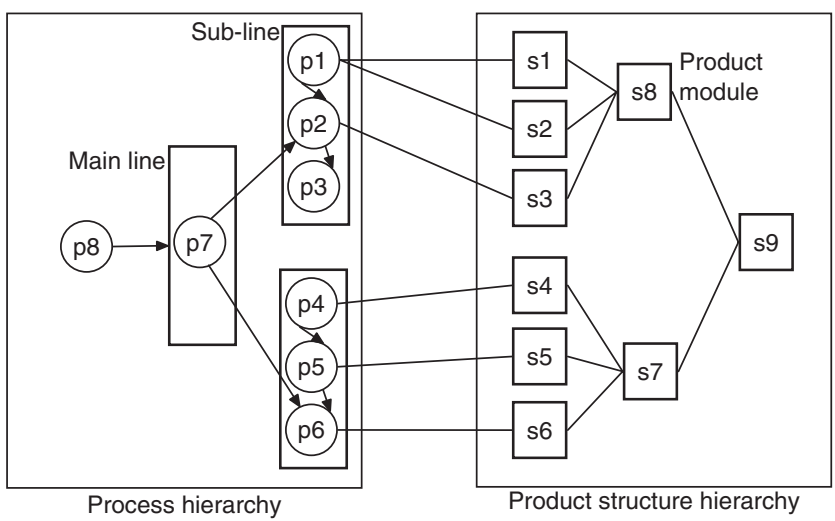

Figure 3. Modularization in production: multiple hierarchies.

and constraints. This approach is interesting, since it defines some of the fundamental concepts used in the MANDATE approach.

\subsection{Product, process, resource: an ontology- based approach}

An important ontology developed to date from the standpoint of scheduling and resource allocation is the OZONE scheduling ontology project. The OZONE ontology is the result of considerable prior experience in building planning and scheduling systems, in application domains ranging from manufacturing production scheduling to space mission planning to military deployment and aero-medical evacuation (re-)planning. For Becker and Smith [8], the class library design and implementation underlying the OZONE framework (and the ontology which provides its conceptual foundation) have followed from retrospective analysis of these scheduling domains and systems, together with application of object-oriented analysis and design principles.

The OZONE ontology adopts an activity-centered modeling viewpoint turned towards constraint-based scheduling. Scheduling is defined as a process of feasibly synchronizing the use of 'resources' by 'activities' to satisfy 'demands' over time, and application problems are described in terms of this abstract domain model. Figure 4 illustrates the base concepts involved and their structural relationships. A 'demand' is an input request for one or more 'products', which designate the 'goods' or 'services' required. More generally, the 'demand' is the interface that allows an external client to state the objective to be achieved as well as certain user specified restrictions and/or preferences on this objective. The objective specified in the 'demand' is the expected output of the system. For example, if a customer orders a computer from a computer manufacturing company, 


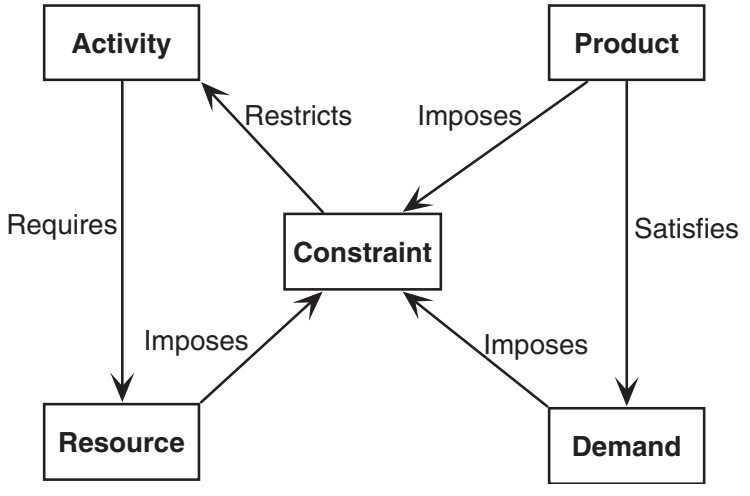

Figure 4. OZONE abstract domain model [8].

the output expected by the user from the company is the computer with the configuration specified in the order. This expected system output is the 'product'. The product can be a physical entity like the computer ordered; or the satisfaction of some conceptual specification like the transfer of the location of an object, or even some more abstract goals that have no actual physical meaning.

The ability to generate the expected output according to specifications is a property of the system. A computer company can assemble only a certain range of configurations and a transportation company can support only certain types of cargo. The different types of objectives that can be accomplished by the system characterize the set of 'products' available to the user of that particular system. In the OZONE ontology, the 'product' entity represents the knowledge required by the scheduler to generate a set of resource allocations over time. A scheduling system does not generate any physical object nor produces any change in the real world. The production and the transportation systems are the entities responsible for the actual accomplishment of the objective. In a scheduling system, the 'product' encodes the internal information about resources and physical characteristics of the process that when combined with the external demand allows the generation of a set of 'resource' requirements over time. These resource requirements are the 'activities'. Therefore, the 'product' can be seen as the template plan for accomplishing a certain goal or a certain set of goals. The 'demand' provides the parameters that maps this prototypical plan into an 'activity' network that when executed would accomplish the specified objective.

The satisfaction of 'demands' centers around the execution of 'activities'. An 'activity' is a process that uses 'resources' to produce goods or provide services. An 'activity' can only be executed if certain conditions, like resource availability, are satisfied. The execution of an 'activity' produces changes in the state of the real world. Notice that although 'products' are 'produced' as a result of the execution of activities, they play a different role in the OZONE ontology. They represent the set of valid objectives that can be specified in a demand; the set of objectives the system knows that can be satisfied with the set of 'resources' available. The 'product' entity acts more as a link connecting 'demands' to 'activities' through 'resources' than a means of describing the result of executing activities. Using the example, the 'product' for the computer manufacturing scheduling system is the process plan for producing the configuration specified. When the order is input into the scheduling system, the 'product' has the information necessary to create a process plan that when executed would produce the required computer. The scheduler only allocates time on the resources specified in the plan.

The use of 'resources' and the execution of 'activities' is restricted by a set of 'constraints'. These 'constraints' can be specified by the 'demand', like release date and due date; can be inherent to the 'product' characteristics, like technological restrictions and design parameters; or can be a result of the 'resource' limitations, like resource capacity, speed, and accuracy.

These five base concepts of the ontology - 'demand', 'activity', 'resource', 'product', and 'constraint' together with the inter-relationships depicted in Figure 4, define an abstract model of a scheduling domain, and a framework for analyzing and describing particular application environments. This framework can be considered as a modular framework, since it is developed on top of this set of five independent concepts.

Associated with each concept definition are terminologies for describing basic properties and capabilities. Properties define attributes or parameters of relevance to specifying an executable scheduling model. The abstract model and its properties are extensible through concept specializations to define more specific models for various sub-domains.

The following sections explore this modularity, through the presentation of the ISO 15531 MANDATE standard. This standard, developed within the framework of the ISO TC 184/SC4 committee, deals with manufacturing management information. This standard currently proposes three information models, related to resource information, to the time, and to flow information. References to 'product' are made external to the models, in order to keep their level of genericity as high as possible.

\section{The ISO 15531 MANDATE standard}

Starting with a short description of the international standardization context leading to the development of the standard, this section gives details about the structure and the different models the standard is made of. 


\subsection{The ISO TC 184/SC4 standardization context}

The work of the SC4 standardization committee includes all the industrial data related to discrete products including, but not limited to: geometric design and tolerance data, material and functional specifications, product differentiation and configuration, process design data, production data (including cost), product support and logistics, life cycle data, quality data, and disposal planning data [9]. It also includes organizational data, such as the relationship between enterprises or the relationship between components of a single enterprise for the purposes of supplier identification. It includes personnel data to the extent of identification of approvals. Specifically excluded are business planning data, such as profit projections, cash flow, etc., and any other personnel data or organizational data. The goal of SC4 is the creation and maintenance of standards that enable the capture of information comprising a computerized product model in a neutral form without loss of completeness and integrity throughout the life cycle of the product.

\subsection{Main features and structure of the standard}

A manufacturing management system manages the flow of materials and products through the whole production chain, from suppliers, through manufacturers, assemblers, to distributors, and sometimes customers. The relations among those partners may be identified and structured in an electronic form with a view to facilitate electronic exchanges. Then, information handled during these exchanges have to be identified, modeled, and represented in such a way that they may be shared by a maximum of partners. From this analysis, three main categories of data related to manufacturing management can be distinguished:

- information related to the management of the time;

- information related to the management of the resources used during the manufacturing processes;

- information related to the management of the manufacturing flows.

MANDATE is an International Standard for the computer-interpretable representation and exchange of industrial manufacturing management data. The nature of the description makes it suitable not only for neutral file exchange, but also as a basis for implementing and sharing manufacturing management databases and archiving. The standard is focused on discrete manufacturing, but not limited to it. The purpose is to facilitate the integration between the numerous industrial applications by means of common, standardized software tools able to represent these three sets of data.

The standard is organized as a series of parts, each published separately. The parts belong to the following series:

(IS: International Standard, WD: Working Draft)

- Manufacturing resources usage management data (3x series):

- ISO IS 15531-31: Resource Information Model: Basic Concepts [10];

- ISO IS 15531-32: Conceptual Model for Resources Usage Management Data [11].

- Manufacturing flow management data (4x series):

- ISO IS 15531-42: Time Model [12];

- ISO IS 15531-43: Data Model for Manufacturing Flow Management [13];

- ISO WD 15531-44: Shop Floor data for Manufacturing Management.

MANDATE Part 1 [14] provides a general overview, specifying the functions of the various series of parts of the standard and the relationships among them. It also specifies the relations between the standard and other related standards. All the MANDATE parts are written using the EXPRESS language (10303-11) [15].

\subsection{The Resource Information Model (ISO 15531-32)}

The conceptual information model for resources usage management data is structured into six logical modules, which are: resource hierarchy (generic, specific, individual resource), resource characteristics (set of information about a resource), resource administration (administrative information), resource status (availability or not of the resource), resource view (specific aggregation of resources), resource representation (physical values), resource configuration, see [11] for a detailed representation of the resource usage management schema.

A resource is the basic element for resource management. Each further detailed description, classification or configuration of resources relates to resource. A resource can be generic, specific, or individual and may in turn be made of a number of other resources. Each resource has characteristics and can also be considered from different viewpoints. It is important to notice that a resource is not a priori related to any given activity. It exists and may be managed before any appointment to any activity. That is typically the case for human resources.

Resource properties are defined by references to external modules, or catalogues, structured by means of the ISO 13584 P-LIB (Part Library) standard [16]. 


\subsection{Data Model for Manufacturing Flow Management (ISO 15531-43)}

This part addresses the modeling of data for the management of manufacturing flows as well as flow controls in a shop floor or in a factory. This manufacturing flow model is provided in the context of various processes that run simultaneously and/or sequentially, providing one or more products and/or components and involving numerous resources. This part (see [13] for a detailed representation) provides a way to model the data needed to manage the multiple complex flows that have to be taken into account between the different manufacturing processes in a factory. That includes products, components, or raw material flows as well as services flows, such as information flows [17]. The main entities of the schema are:

- process: structured set of activities involving various enterprise entities, designed and organized for a given purpose;

- flow: motion of a set of physical or informational objects in space and time.

As for the resource information model, process properties and flow properties are defined by references to external modules, or catalogues, structured according to the ISO 13584 P-LIB standard.

\subsection{Time Model (ISO 15531-42)}

The time model is made of two 'schemas', with the meaning of the EXPRESS representation: the domain property schema, providing a generic topological structure applicable to any one-dimensional domain, and the time domain schema, instanciation of the previous generic structure to the time. The time domain schema is presented here.

The time schema provides the definition of concepts related to the time representation, needed by software applications mainly dealing with scheduling and manufacturing management operations. It enables multiple representations of time domains, intervals of time, points in time, and time units. The time domain schema is represented in Figure 7.

For the management of industrial manufacturing systems, an assignment of a point in time to an event occurrence is necessary to enable the observation and comparison of points in time characteristic of the system.

Since the time domain and all its related subcategories only define different categories of sets of points, another entity is needed to enable the consideration of the length, the duration of a period in time: this concept is provided by the entity interval of time, whose duration is given by the type of value of point or interval of time entity. The concept of frequency of events is an important feature of this model, since it enables the characterization of the intervals of time separating event occurrences. Transformation rules between two time domains are provided through the entity time domain relation. These rules can be expressed either in terms of time unit or in terms of change of origin (translation).

Time is defined by a time domain containing a sequence of points in time. A point in time is defined by a selected location on the time axis, through the use of a time unit. A time unit is used to measure the duration in the related time domain. A time domain is defined as follows:

$$
\begin{aligned}
(T, \leq) \text { with } \quad T & =\text { set of points in time, } \\
\leq & =\text { complete order - relation on } T .
\end{aligned}
$$

This definition allows for both continuous and time domains considered by this standard, see [12] for a detailed representation of the model.

In the following section, the role of the standard in industrial production management systems is discussedz through its specificity in terms of products, but also in terms of processes and resources management.

\section{MANDATE in the industry}

In terms of industrial maturity, MANDATE is a 'young' standard, whose development started recently and whose parts have not reached the IS status (necessary for sake of stability) at the same time. For this reason, the different models proposed by the standard have not been implemented at the same time. In this section, the implementation of the resource information model is discussed, alone, then alongside with the other models in a MRPII structure. Then the manufacturing scenario developed within the framework of the project is presented.

\subsection{Modularity and genericity of the standard}

The standard can be considered as providing two levels of modularity: on the one hand, it is modular since each part is independent from the other ones. On the other hand, its modularity comes from the fact that all the references to given products, resources, processes are made external, and thus are not pre-defined in the standard: this standard is thus more generic and independent from any specific kind of product, resource, and process. However, it is easy to specialize to a specific usage. 
- Modularity in terms of products targeted by production management systems (PMS): MANDATE does not apply to a specific 'product'. The standard makes use as far as possible of product models defined by the ISO 10303 STEP standard. This concept of 'product', as it is defined in ISO 10303-1 [18] is a powerful and original feature of the standard, compared to other exchange standards. The objective is to provide a mechanism capable of describing product data, throughout the life cycle of a product, independent from any particular system and applicable to any kind of product. ISO 10303 STEP takes a product-oriented view of manufacturing, while MANDATE deals with the data defining the processes, within the overall entreprise.

- Modularity in terms of processes and resources needed by the PMS: As for products, MANDATE does not target specific processes and resources, either industrial or not. All information, characteristics, features, catalogue data related to processes and resources are considered as external to the standard; they are provided by the ISO 13584 Parts Library representation: whose purpose is to specify a form for the unambiguous representation and exchange of computer-interpretable parts library information by grouping the common features of the parts. This form is independent of any particular computer system, allows for any kind of part representation category, and enables consistent implementations across multiple applications and systems. The standard permits different implementation technologies to be used for storing, accessing, transferring, and archiving parts library data.

- Use of ISO 13584 (P-LIB) libraries: in the domain of component libraries, the P-LIB (ISO 13584) standard separates the information about the structure of a parts library, from the information about each part or family of parts that belongs to the parts library. As such, the standard is well suited to the management of the commonalities of the parts. P-LIB also makes use of the EXPRESS language to specify the information about the structure of a parts library. It allows the information about each part, or each family of parts belonging to a parts library to be specified by different standards. The conformance testing of the implementations will use the same methodology and framework as defined in the ISO 10303 standard.

- Genericity of the standard: MANDATE provides a generic approach of some of the main concepts used in production management systems. As such, the standard cannot be used alone, but specialized through the instanciation of constructs written in terms of resources, flows, and time developed in the parts 32, 42, and 43. This instanciation is made through links provided by the standard to external component libraries: resource libraries, flow libraries, process libraries. Those libraries generally exist within the companies, however they are most of the time implicit, the knowledge embedded is not often explicitely formalized nor expressed under a specific (electronic) format - they can also be partially developed and implemented, often in that case closely related to a specific software tool devoted to a specific task, thus, and most of the time represented using a proprietary format not transferable. This knowledge contains the history, the know-how of the enterprise, also the added value and the skill of the company. The development of such P-LIB based libraries, expressed and stored independently from any particular software tool, is a powerful way to record and to structure their know-how, their activity, their skill. It is also a way to make this knowledge modular, thus more adaptable to new situations companies may face in the future. It is also a way to improve interoperability among the different software applications used throughout the different departments and/or plants of the companies.

The modular structure of the MANDATE standard enables its use in different contexts of a manufacturing enterprise. In the section below, the resource information model is applied to the management of a machining cell. Beyond the environment of the shop floor, at a larger scale, a manufacturing resource planning (MRPII) approach makes use of all the models proposed by the standard.

\subsection{Resource Information Model}

Machine tools, fixtures, cutting tools, manufacturing personnel, pallets, transfer devices, coolant, etc. can all be considered as being manufacturing resources [11]. The combination of a range of such resources provides a manufacturing capability which can be assessed in terms of its usage. Resource information can be combined in many different ways dependent on the purpose for which it is needed.

Many combinations of resource can be considered. Another example is provided by an assembly shop with a range of assembly machines each of which can perform different assembly operations. The combination of these machines and the characteristics of each influence the overall potential usage of the shop. The shop itself can be considered to be a resource with a set of characteristics as can each of the cells within the shop and each of the machine stations within each cell.

The machine in the shop is an example of a specific resource. It has a set of characteristics, such as capacity and capability. However if one considers it to be a machine supplier's machine it is therefore not an actual resource which exists within the business. This illustrates the difference between a specific resource and an 
individual resource. An individual resource can be considered as a resource occurrence which will have some status at some point in time.

The resource characteristic is the means by which sets of values are assigned to resources. Each resource is defined by a set of characteristics and each characteristic has a representation and a grouping. The representation of the characteristic simply relates to quantitative or qualitative values. However, the representation is also classified in terms of whether the value is a proposed value, a required value, or a value which has been realized. It is recognized that additional classifications may be required and this can be achieved through the link from library properties. The grouping of the resource characteristic is effectively another classification of the characteristic in terms of whether it is concerned with the administration of the resource, the capacity, or capability of the resource or the constitution of the resource.

While the recursive resource definition enables flexible resource groups to be defined a resource may be viewed from a number of different perspectives. For example, a factory may be considered to be a resource which can be broken down into shops, cells, and stations. However, views of the personnel within the factory could be defined; views of a particular set of machine types could be defined; views of the tooling for tool management purposes could be defined.

The resource view allows views of resources to be defined either as a user-defined view or following the DIN 4000-1 [19] approach of specifying resources by a tabular layout of article characteristics.

\subsection{Use of the different MANDATE models}

Globalization has left many manufacturing and service-oriented companies with the option of pursuing world's best practices or perish. Many companies now realize the need for the development of world class systems and methodologies, as well as acquiring the 'productivity tool' that will let them be in a commercial position to offer competitive manufacturing resource planning that assures customers of quality goods and services and compliance with international quality requirements on different industry fields [20]. In the competitive business environment of the twenty-first century, the development of a manufacturing strategy, as 'a collective pattern of decisions that acts upon the formulation and deployment of manufacturing resources' (APICS Dictionary, see [21]), becomes more and more important.

In this context, the MANDATE models bring an important contribution to the MRPII information modeling approach. The APICS Dictionary (APICS) defines MRPII as 'a method for the effective planning of all resources of a manufacturing company.
Ideally, it addresses operational planning in units, financial planning in dollars, and has a simulation capability to answer what-if questions. It is made up of a variety of processes, each linked together: business planning, production planning (sales and operations planning), master production scheduling, material requirements planning, capacity requirements planning, and the execution support systems for capacity and material. Output from these systems is integrated with financial reports such as the business plan, purchase commitment report, shipping budget, and inventory projections in dollars. Manufacturing resource planning is a direct outgrowth and extension of closed-loop MRP'.

Figure 5 makes appear the main functions of the MRPII approach: the MANDATE models can be used to structure the information and the data exchanged at the interfaces between the different functions (or 'boxes') of the schema. The time model does not explicitly appear on this schema, its role is implicit since it provides the mandatory sequencing of all the activities of the production cycle.

\subsection{Manufacturing scenario}

A manufacturing scenario has been developed within the framework of the MANDATE project. The objective of the scenario was, through a complete description of the information related to the manufacturing management of an industrial product, to show a way of using and relating together the different parts of the standard. However, at the time of completion of the scenario, the part 43 about process and flow management was not yet finalized, and it has been decided to focus the scenario mainly on the use of the resource information model. Another objective was to show the possible links with other standards, along with the connection points and the roles of these links. The example was based on a real manufacturing test case of a commonly used industrial product. For each function of the production process described, the scenario provides the structure of the data sets corresponding to the inputs, outputs, constraints (if needed) of the function. The product selected is a particular type of gear wheel, called gw_1-c. The product of the example is defined by a set of engineering and manufacturing data, described in the following files:

*PROD_ITEM file: information related to products as items: main file for all items, products, whether purchased, manufactured, managed in stock;

* PROD_STRUCT file: product structures, used mainly for planning and product costing. The result is a product structure containing necessary information for planning, costing, and manufacturing;

* PROD_ROUTE file: information necessary for routing, bill of labor. 


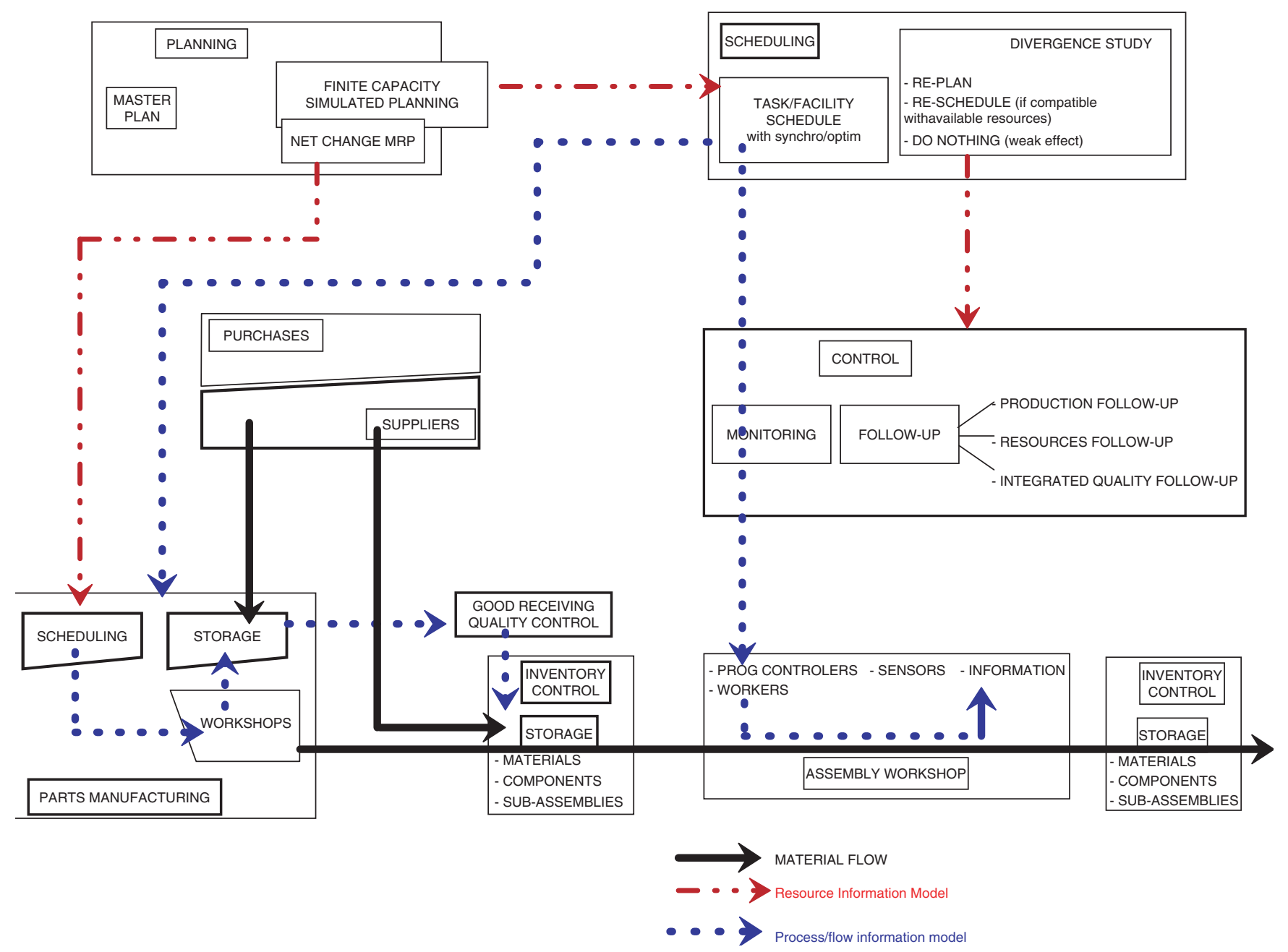

Figure 5. Merging of the different MANDATE information models in a MRPII environment.

Table 1. Excerpt of the PROD ROUTE table: information necessary for routing, for the bill of labor.

\begin{tabular}{lll}
\hline Field_num & Field_name & Text \\
\hline 1 & prodro_com & Company \\
2 & prodro_facil & Facility \\
3 & prodro_num & Product number \\
4 & prodro_str_type & Product structure type \\
5 & prodro_oper_num & Operation number \\
6 & prodro_sequ_num & Sequence number \\
7 & prodro_fro_date & From date \\
8 & prodro_to_date & To date \\
9 & prodro_work-center & Work center \\
& & (area, or technical group) \\
10 & prodro_oper_descr & Operation description \\
\hline
\end{tabular}

Table 1 shows an excerpt of the prod_route file.

The objective of the scenario was to map the concepts from the industrial example to the entities of the resource information model: the correspondences between the content of the gear wheel example file and the RIM names (ISO 15531-32 part); input to the ISO 115531-42 time model part are also mentioned. An excerpt of the result is represented in Table 2.
The parsing of the example coming from the manufacturing scenario on the MANDATE models was very interesting, since it provided the opportunity to test the models against the case of a real product given with its definition and the necessary manufacturing capabilities, capacities, and constraints. The first step of the work was to identify the set of data relevant to the resource information model and to the time model: this task was not a trivial one, since the data were provided into three files (tables) related to the product taken as an item (47 fields in the table), the structure of the product (36 fields), the route of the product (36 fields). Those tables were generated by the production management application of the company. As they were provided, it was not easy to identify the different attributes, key and non-key and to highlight the dependencies between the attributes and with the tables. Since the tables contained both information relevant to product management and to manufacturing management, the second stage was to put apart information related to product, in order to focus on manufacturing management information: example: from the product_structure table: product 
Table 2. Correspondences between the content of the gear wheel example file and the RIM.

\begin{tabular}{rlll}
\hline Num & Field_name & Text & RIM corresp. Name or other \\
\hline 9 & Prodro_work-center & Work center (area, or technical group) & resource_group \\
10 & Prodro_oper_descr & Operation description & resource_view, resource_user_defined_view \\
11 & Prodro_text1 & Text - line 1 & \\
12 & Prodro_text2 & Text - line 2 & \\
13 & prodro_alter_oper & Alternate operation & resource_view \\
14 & prodro_oper_activ & Operation activities (if used) & \\
15 & prodro_phantom_oper & Phantom operation method & resource_status \\
17 & prodro_fixedt & Fixed time & point_in_time \\
18 & prodro_setupt & Setup time & point_in_time \\
19 & prodro_runt & Run time & point_in_time \\
20 & prodro_time_qty & Time quantity & point_in_time \\
21 & prodro_planw_setup & Planned number of workers - setup & resource_characteristic_group \\
22 & prodro_planw_runt & Planned number of workers - run time & resource_user_defined_view \\
23 & prodro_plan_mach & Planned number of machines & resource_user_defined_view \\
28 & prodro_init_weight & Initial weight & tabular_layout_of_resource_characteristics \\
29 & prodro_lab_skill & Labor skill & resource_capability \\
31 & prodro_tool_num & Tool number & resource_name \\
32 & prodro_lab_ticket_num & Number of labor tickets & resource_characteristic \\
34 & prodro_leadt_offset & Lead time offset & interval_of_time \\
35 & prodro_production_days & Production days & point_in_time \\
36 & Prodro_supplier_num & Supplier number & tabular_layout_of_resource_characteristics \\
\hline
\end{tabular}

number, product structure type, product text can be provided by external references to corresponding attributes of the entity 'product' from the ISO 10303 STEP standard. Another point discovered in the example was the fact that sometimes, elements of information were put into attributes when it was possible to calculate this information (e.g., weight of a sub-assembly, number of operations). Sometimes also, the meaning of the attributes, and the format used were ambiguous.

Of course, one of the big difficulties met was to be able to separate the 'corporate' information, specific to the company, from the common information needed by production management systems, in order to translate only the information relevant to manufacturing management into elements of the MANDATE models. This problem will disappear as the external libraries, containing all this information are made available.

As a matter of result, once restricted to manufacturing management information, a good correlation was found between the needs in terms of information coming from the real test case and the entities of the models: both for the resource information model and the time model. However, the difficulties experimented came out from the lack of available external references, since no catalogues were available for the different properties and features about products, resources, and flows.

This problem is discussed in the last section.

\subsection{Discussion - problems arising}

Standards basically developed for manufacturing integration and able to capture the enterprise semantics apply to the following domains: data integration (among which: ISO 10303 STEP, ISO 15531 MANDATE, ISO 13584 PLIB), enterprise models and architecture, communication, interfaces, and translation. The main features of those standards are:

- For STEP (ISO 10303): product data modeling, based on a specific modeling language EXPRESS (ISO 10303-11) and on 'integrated resources' (parts $4 \mathrm{x}$ series), application protocols make use of integrated resources for specific area.

- For PLIB (ISO 13584): design and provision of parts library and components, implemented (particularly in Japan), well suited to e-business, now at the stage of development of specific standardized catalogues (fastener, cutting tools, measurement devices) often in joint collaboration with IEC, used by other standards to specify constructs or partial models (MANDATE).

Standardization committees are more and more oriented towards the standardization of the semantics, considered as a basic need to share and exchange information, data, and knowledge. On the other hand, product data engineering is now mature and the users in the enterprise are more and more interested in the standardization of their systems and architectures.

However, a question remains about the coverage of the different standards, through their models, their representations and, first of all, the vocabulary used: the authors presented in Section 2 of this study the result of the analysis of the terms 'product', 'process', and 'resource' made by Michel [5], entitled 'terminology extracted from some manufacturing and modeling 
related standards', where he compares the meaning of the main terms used in manufacturing management. It is easy to see that the meaning, the semantic embedded into those definitions is not the same... although all of them refer to the same 'concept'. However, in terms of interoperability among software tools, inconsistencies, gaps, appear, thus making the translation impossible, or at least of poor quality.

How to solve this problem Several approaches are possible. Among them, the use of translation languages based on ontological representations of the concepts (such as the ISO 18629 PSL language [22]), and the use of transformation methods is worth mentioning. In the following section, model transformation methods are presented, as they have been studied within the framework of the INTEROP project.

\section{Model integration: need for model transformation methods}

Enterprises today face many challenges related to the lack of interoperability. Enterprise applications and software systems need to be interoperable in order to achieve seamless business across organizational boundaries and thus realize virtual networked organizations [23]. Model-driven development (MDD), and in particular OMG's model-driven architecture $^{\circledR}$ $\left(\mathrm{MDA}^{\circledR} 1\right)$ [24], is emerging as the state of practice for developing modern enterprise applications and software systems. The MDD paradigm provides a better way of addressing and solving interoperability issues compared to earlier non-modeling approaches. However, developing correct and useful models to address interoperability is not an easy task!

In this section, some results are presented from the INTEROP EC project in defining an interoperability framework for model-driven development of enterprise applications and software systems. The framework provides a foundation, consisting of a set of reference models, describing how to apply MDD in software engineering disciplines in order to support the business interoperability needs of an enterprise.

\subsection{Presentation of the INTEROP Project}

INTEROP (IST-508011) is a network of excellence (NoE) supported by the European Commission for a three and a half year period, starting from 2003. INTEROP is aimed at creating conditions of an innovative and competitive research in the domain of interoperability for enterprise applications and software [25].
The integration will be achieved by the end of the three-year project duration. Meanwhile, INTEROP spreading of excellence activities should ensure the fertilization of the largest research community as well as IT providers and users, to provide a durable virtual lab on interoperability beyond the EU-funded period.

The objective of the INTEROP portal is to increase awareness on interoperability, to keep informed on the research results developed within the consortium but also to share experiences on collective methods of work. It has been designed to provide a single point of access to all relevant information and applications, functioning as an access to this interoperability communities of interest and practice.

The following sections focus on the work done by the Task Group 2 (TG2: MDI - model driven interoperability), since some of the authors of this study, within the framework of this project, have particularly worked on model transformation methods.

\subsection{Model Driven Interoperability}

The aim of the work is to analyze the current situation and to bring solutions in the domain of model driven interoperability (MDI). The approach combines results coming from three domains: enterprise modeling, ontology and architecture and platform - together with the domain of enterprise software applications (ESA).

The need for interoperability between ESA appears in various situations:

- introduction of a new type of ESA in the enterprise and connection to the existing one,

- connection of ESA between several enterprises, merging of enterprises and integration of legacy software, and similar situations.

In the model driven architecture (MDA) methodology, modeling is made according to three points of view corresponding to three conceptual levels:

- Computation Independent Model (CIM): representing system requirements in the environment in which it is going to operate, for business models with a holistic point of view about the enterprise.

- Platform Independent Model (PIM): modeling system functionality but without defining how and on which platform it will be implemented, focused on information and seen from a computational point of view.

- Platform Specific Model (PSM): PIM is transformed into a platform specific model according to a

${ }^{1}$ Model Driven Architecture ${ }^{\circledR}$ and MDA $^{\circledR}$ are registered trademarks of the Object Management Group. 


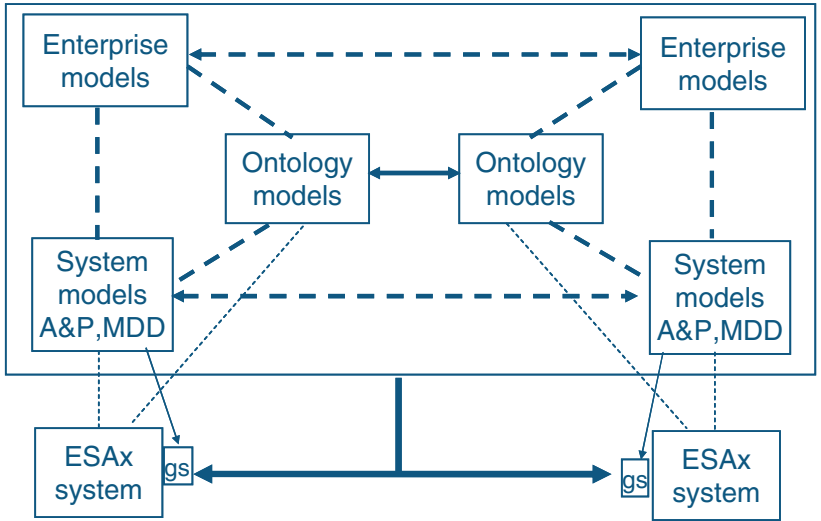

Figure 6. Model driven interoperability. A\&P: Architecture \& Platform MDD: Model Driven Development ESA: Enterprise Software Applications.

selected platform, focusing on a technological point of view.

In a model-driven development process, it is possible to develop an extensive set of different interrelated models at different abstraction levels, ranging from business models, requirements models, and design models to deployment models and code, code generation, and model synchronization. Based on the initial objectives of the TG2, three tasks have been identified: model establishment and system configuration (M), model interoperability (MI), and MDI. The focus of the MDI task is to show how the ESA system interoperability can be driven by the developed interoperability models. One goal is to be able to generate appropriate mapping software (gs) that can support mapping between external interoperable models, and internal representations - making easy the internal mapping configuration.

The first work planned by the TG2 was to create different enterprise models from a real case study in order to establish relevant models and relationships between those models. First, relationships were defined among different enterprise models and particularly among those modeled at the different levels of abstraction, CIM, PIM, and PSM. Practical experiences of enterprise model analyses have been carried out on a specific real case study provided by a partner of the project. For the purpose of the task, the following enterprise modeling languages were analyzed:

- GRAI: to model all dimensions of the enterprise, mainly focusing on the decisional dimension [26];

- IDEF: to model all dimensions of the enterprise, mainly focusing on the process dimension [27];

- UML: to analyze its use for enterprise modeling $[28,29]$.

An important contribution of the task has been to provide a first approach on a model transformation process, taking into consideration the semantics of the models.

\subsection{Mappings and model transformations}

An important work to be carried out was to define mappings between the different meta-models in order to perform transformations at different levels:

- Horizontally: in order to perform transformations from one CIM model into another;

- Vertically: to transform CIM models into PIM, then PSM.

These transformations cannot be performed automatically without adding semantic annotations to the source models. This semantics enrichment can be performed through an ontological point of view, represented in Figure 6.

In this figure, enterprise models on the upper left corner are put in correspondence with system models (bottom left corner) through ontology models embedding the semantics specific to those enterprises. Same analysis is made for other enterprise models represented in the upper right corner of the schema: they are put in relation to other system models (bottom right corner) through other ontology models. In the MDI approach, correspondences are then developed between the different ontology models represented in the middle of the schema.

The feasibility of model transformations from CIM to PIM and then to PSM has been demonstrated through the use of a transformation language, associated to the corresponding transformation tool:

- Atlas Transformation Language (ATL) developed by the ATLAS INRIA and LINA Research Group in conformance with OMG. It is a model transformation language specified both as a meta-model and as a specific textual concrete syntax, hybrid of declarative and imperative languages based on OCL [30];

- ATL Development Tools (ADT): Integrated development environment (IDE) developed for ATL on top of eclipse [31]. It uses the eclipse modeling framework (EMF) to handle graphical models, to serialize and de-serialize them, as well as to navigate and to modify them.

A first pilot test of this IDE was performed and it resulted from the analysis that this kind of tool could be used at the following levels:

- to build transformations between enterprise models expressed with different formalisms (GRAI, IDEF0, $\mathrm{UML}, \ldots$ ); 


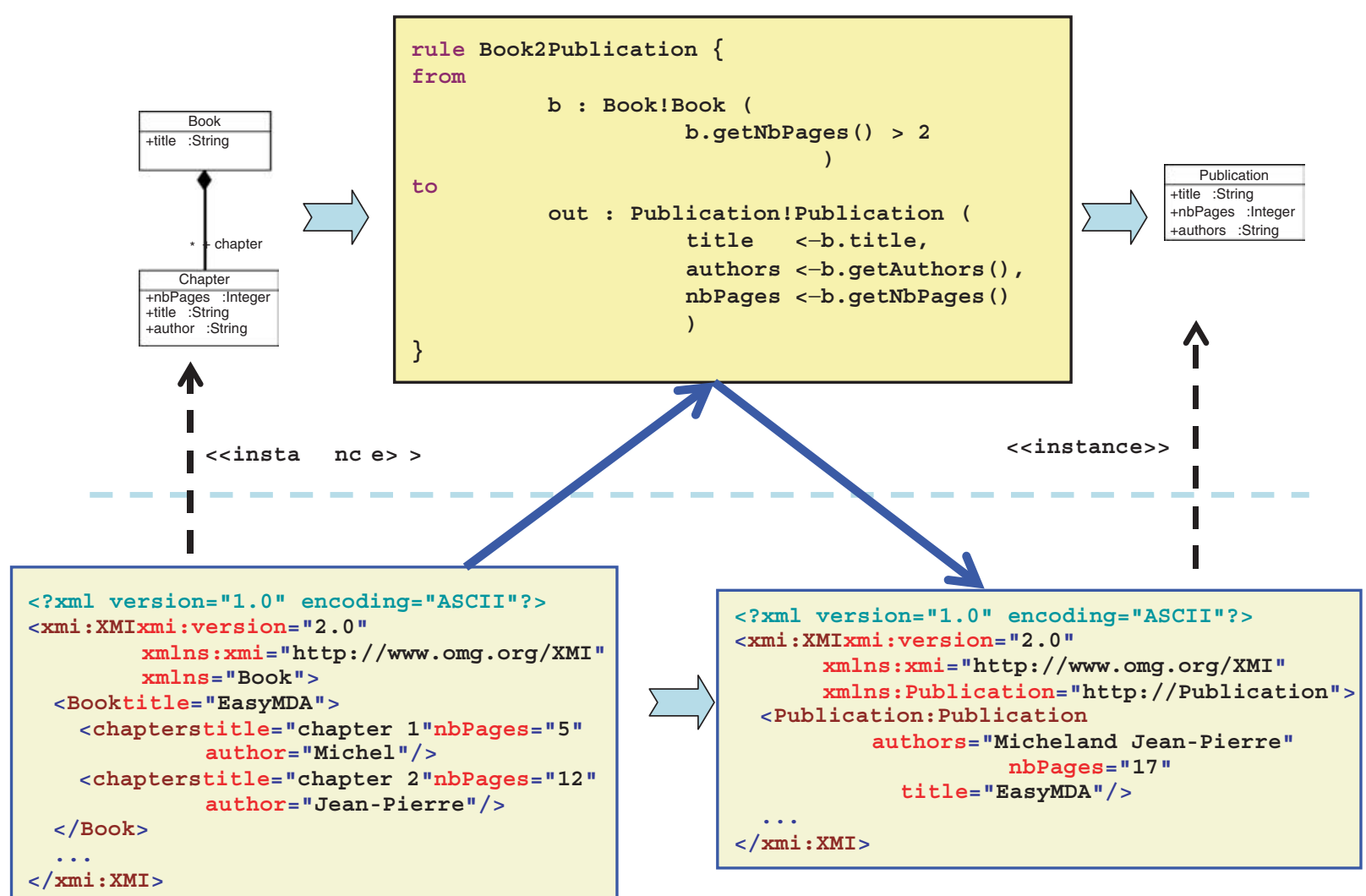

Figure 7. Working framework of ADT.

- to build transformations between different levels (CIM, PIM, and PSM).

To perform a transformation with the ATL tool, four elements are needed (see Figure 7): source (meta)-model, target (meta)-model, mapping between source and target expressed in ATL, and input model which instantiated the source (meta)-model.

The transformation can then be performed to get the target model, instance of the target (meta)-model. Mappings between formalisms have to be defined at the meta-modeling level. If the mapping is not a oneto-one mapping between one source concept and one target concept but a one-to-many mapping, the transformation needs additional semantic annotations or semantic support to produce the target concepts. These annotations must provide information about the target concept that has been chosen. For instance, within the UML modeling domain, these annotations can be provided by tagged values or stereotyped notes attached to modeling elements.

Those transformation principles have been applied to the transformation from GRAI to UML at the computation independent model (CIM) level. The full transformation process is presented in the deliverable TG2.1 of the INTEROP Project [32].

In the domain of manufacturing engineering, and given the terminology issues represented in Figure 1 and the diversity of the information models available, one can see here a strong indication of the potential for MDA approaches to provide improved support for semantic interoperability. Investigations are being made by the authors to apply model driven methods to this area.

\section{Discussion - issues}

In this study the authors have discussed a way of managing modularity in production management systems through the use of international standards. The first section presented a modular approach to production management, the modularity being here based on a separate processing of the concepts of 'product', 'process' and 'resources'. The OZONE ontology was also presented, since the project proposes an interesting focus on scheduling management seen from this point of view. This modularity is a fundamental feature of the MANDATE standard presented in the following sections. MANDATE is a new emerging standard in the domain of manufacturing management, the physical implementation of the concepts proposed is just starting and a manufacturing scenario is presented.

Numerous standards do exist at the different levels of the production management systems, but their joint use highlights some problems, among which: the lack of 
compatibility of the information models, the vocabulary used is not defined in the same way even though the terms used are the same: ontology-based approaches are sometimes necessary to find the common 'essence' of the information handled, further they can be integrated in software interfaces, making easier to convey a higher level of semantics in the exchanges. This study presented one of those approaches, proposed by the INTEROP EC funded project.

It is interesting to analyze the trends and the role of international standards for intelligent collaboration and integration in manufacturing. The needs: to improve productivity and efficiency and to reduce the manufacturing and the time to market life cycles. To achieve that it is necessary to avoid failures in communication flows, to improve accuracy of data processing and associated manufacturing processes, then to share and to exchange manufacturing data and models inside the enterprise as well as with its environment in an accurate way [33].

Semantic versus technological integration: to share or to exchange data and models between application A and $\mathrm{B}$ two conditions are required: the semantics carried out by data and models of the applications $\mathrm{A}$ and $\mathrm{B}$ must have a least a part in common; the technological tools used to exchange this common semantics and/or to share it must be compatible or interoperable.

Technological tools include: data management and data access tools (DBMS, programming languages, query languages...), communication tools (LAN, WAN, EDI, etc.). Are of course included WWW technologies and services (HTML, XML, OWL, etc). Technology is always evolving and new standards or new editions of standards appear every time. The main evolutions are more and more linked to the number and diversity of new technological tools appearing about the web and the e-business.

In this domain, the main trend is the recent and permanent growing of standards that enable the enterprise to preserve and re-use the semantics included in its applications.

Intelligent collaboration and integration in manufacturing are characterized by:

- The level of integration (physical, application, business);

- The way of integration (top-down or bottom-up approach);

- The expected results (full integration, unification, federation):

- The tools and methods implemented (enterprise organization integration, data integration, communication integration, integration through interfaces and translations).
The expected results coming out from the integration process are:

- In the case of full integration (e.g., proprietary software) the standard is the software itself;

- In the case of unification (e.g., Windows, Office, Unix) the integration makes use of standardized components (constructs, partial models) and standardized interfaces;

- In the case of federation (e.g., legacy software built around various products) the impact of standardization is very poor. Some standardized interfaces and communication standards may be used as well as standardized translators.

Standards appear as particularly useful in the integration by unification, since, when they are used together, they provide standardized components (enterprise models, partial models, constructs) that are shareable, re-usable, and interoperable. They also convey and preserve the common part of the semantics included in the various enterprise (and inter-enterprise) applications. It is the reason why it is possible to say that the industrial use of MANDATE standard will develop as the physical instanciation of P-LIB catalogues will progress further. This can take time, however the return on investment (ROI) will be substantial, provided that all the information about products, resources, and flows is under an electronic format. Today, MANDATE provides the integration methodology applicable to production management systems.

The methods for integration can be grouped into three categories, which are:

- Data integration (integration through data models). This form of integration is addressed by the standards developed in ISO TC184/SC4 (industrial data);

- Organization integration (integration based on enterprise models, process models, decisional models): mainly addressed by standards developed in ISO TC184/SC5 (systems architecture and communications);

- Communication integration (integration based on network communication models and tools): also addressed by standards developed in ISO TC184/ SC5 but IEC SC65 also addresses the same domain with another point of view.

As a matter of conclusion to this work, a recent US study, made by NIST, highlighted that the use of the ISO 10303 STEP standard presently saves more than 120 million US per year and will save 900 million US per year in 2010 in the US aerospace, automotive and shipbuilding industry $[34,35]$. 


\section{Acknowledgments}

The work reported in the section 5 of this study has been conducted within the framework of the INTEROP Project, Network of Excellence supported by the European Commission, under the contract $\mathrm{N}^{\circ}$ IST508011. The authors would like to acknowledge the support of the INTEROP Community for the work presented here.

\section{References}

1. Frankovic, B., Budinska, L. and Dang, T.T. (2002). Creation of Ontology for Planning and Scheduling, 3rd International Symposium of Hungarian Researchers on Computational Intelligence, Magyar Kutatók 3. Nemzetközi Szimpóziuma, Budapest, Hungary.

2. Takeishi, A. and Fujimoto, T. (2001). Modularization in the Auto Industry: Interlinked Multiple Hierarchies of Product, Production and Supplier Systems, Int. Journal of Automotive Technique and Management, 1(4): 379-396.

3. Young, R.I.M., Guerra, D., Gunendran, G., Das, B.P., Cochran, S. and Cutting-Decelle, A.F. (2004). Sharing Manufacturing Information and Knowledge in Design Decision Support, In: Bramley, A., Brissaud, D., Coutellier, D. and McMahon, C. (eds), Advances in Integrated Design and Manufacturing in Mechanical Engineering, Pub Springer, 2005, pp. 173-188. ISBN 1-4020-3481-4.

4. Ray, S.R. and Jones, A.T. (2003). Manufacturing Interoperability, Concurrent Engineering: Enhanced Interoperable System, Proceedings of the $10^{\text {th }}$ ISPE International Conference, Madeira Island, Portugal, 26-30 July 2003, 535-540.

5. Michel, J.J. (2005). Terminology Extracted from Some Manufacturing and Modelling Related Standards, CEN/ TC 310 N1119R2.

6. Krause, F.L., Kimura, F., Kjellberg, T. and Lu, S.C.Y. (1993). Product Modelling, Annals of CIRP 42/2 695-12.

7. Young, R.I.M., Gunendran, A.G., Cutting-Decelle, A.F. and Gruninger, M. (2006). Manufacturing Knowledge Sharing in PLM: A Progression towards the Use of Heavy Weight Ontologies, IJPR Journal, 45(7): 1505-1519.

8. Becker, M.A. and Smith, S.F. (1997). An Ontology for Multi-modal Transportation Planning and Scheduling, CMU-RI-TR-98-15.

9. http://www.tc184-sc4.org/ (2006).

10. Industrial Automation Systems and Integration - Industrial Manufacturing Management Data - Resource Usage Management Data: Resource Information Model: Basic Concepts: Part 31, ISO TC184/SC4, ISO IS 15531-31, 2004.

11. Industrial Automation Systems and Integration - Industrial Manufacturing Management Data - Resource Usage Management Data: Conceptual Model for Resources Usage Management Data: Part 32, ISO TC184/SC4, ISO IS 15531-32, 2004.

12. Industrial Automation Systems and Integration - Industrial Manufacturing Management Data - Time Model: Part 42, ISO TC184/SC4, ISO IS 15531-42, 2005.

13. Industrial Automation Systems and Integration - Industrial Manufacturing Management Data - Manufacturing Flow Management Data: Data Model for Manufacturing
Flow Management: Part 43, ISO TC184/SC4, ISO IS 15531-43, 2006.

14. Industrial Automation Systems and Integration - Industrial Manufacturing Management Data - General Overview: Part 1, ISO TC184/SC4, ISO IS 15531-1, 2004.

15. Industrial Automation Systems and Integration - Product Data Representation and Exchange - Part 11: Description Methods: The EXPRESS Language Reference Manual, ISO 10303-11, 1994.

16. Industrial Automation Systems and Integration - Parts Library - Conceptual Descriptions - Part 1: Overview and Fundamental Principles, ISO 13584-1, 1997.

17. Cutting-Decelle, A.F., Young, R.I.M., Pouchard, L.C., Bourey, J.P. and Michel, J.J. (2006). A Standardised Data Model for Process and Flow Management: ISO 15535-43 A Step Towards CE in Manufacturing, International Conference CE 06, Sophia-Antipolis.

18. Industrial Automation Systems and Integration - Product Data Representation and Exchange - Part 1: Overview and Fundamental Principles, ISO 10303-1 (1994).

19. Din 4000-1 (1992). Sachmerkmal-Leisten, Begriffe und Grundsätze, Beuth-Verlag, Berlin.

20. Salazar, M.E. (2006). http://www.plant-maintenance.com/ articles/ERP_concepts.shtml

21. Blackstone, Jr., John, H. and Cox, III and James, F. (2005). APICS Dictionary, 11th edn, Alexandria, VA: APICS.

22. Industrial Automation Systems and Integration - Process Specification Language, ISO 18629-1, 2004.

23. Elvesæter, B. Hahn, A. Berre, A.J. and Neple, T. (2005). Towards an Interoperability Framework for ModelDriven Development of Software Systems, INTEROP ESA05 Conference, Geneva.

24. Object Management Group, OMG. (2003). MDA Guide Version 1.0.1, Object Management Group omg/2003-06-01.

25. www.interop-noe.org (2006).

26. Doumeingts, G., Vallespir, B. and Chen, D. (1998). Grai Grid Decisional Modelling, In Bernus, P., Mertins, K. and Schmith, G. (eds), Handbook on Architecture of Information Systems, Springer-Verlag.

27. IDEF (2006). Integrated DEFinition Methods, http:// www.idef.com/.

28. Object Management Group, OMG. (2003). Unified Modeling Language (UML) Specification: Infrastructure, version 2.0, OMG Adopted specification ptc/03-09-15 edition.

29. Object Management Group, OMG. (2004). Unified Modeling Language (UML) Specification: Superstructure, version 2.0., document: ptc/04-10-02 (convenience document) edition.

30. http://www.sciences.univ-nantes.fr/lina/atl/ (2006).

31. http://www.eclipse.org/ (2006).

32. Doumeingts, G., Berre, A., Bourey, J.P. and Grangel, R. (2005). Report on Model Establishment, INTEROP Project, Deliverable DTG2.1.

33. Michel, J.J. (2004). Trends and Role of International Standards for Intelligent Collaboration and Integration in Manufacturing, Intelligent Collaboration in the Supply Chain, Workshop, University of Loughborough.

34. NIST (2002). Economic Impact Assessment of the International Standard for the Exchange of Product Model Data (STEP) in Transportation Equipment Industries, Planning Report \#02-5.

35. NIST (1999). Interoperability Cost Analysis of the US Automotive Supply Chain, Planning Report \#99-1. 


\section{Anne-Françoise Cutting-Decelle}

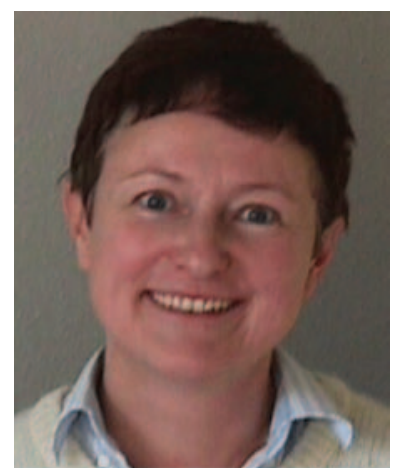

Prof. AF Cutting-Decelle studied at the Ecole Normale Supérieure (Cachan) where she graduated as Agrégée in Civil and Building Engineering in 1979. She got her $\mathrm{PhD}$ at the LGCH laboratory, at the Ecole Supérieure d'Ingénieurs de Chambéry. She is currently professor in Advanced Manufacturing Technologies at the University of Evry, IUT-Department QLIO. Her research activity is done at Ecole Centrale Paris, at the Industrial Engineering Laboratory (LGI). Her research interests are in the fields of formal languages applicable to the representation of heterogeneous data, information, and knowledge managed in construction and manufacturing engineering, and across industrial sectors. This research focuses on the management of the information using the Process Specification Language (PSL, ISO 18629 standard), on the research of correspondences among technical ontologies, and on process grammars able to integrate the logic of industrial process. Another axis of the research is to analyze to what extent the use of formal process languages is possible across two industrial sectors, such as construction and manufacturing engineering. She also works as expert for several standardization committees, in France (AFNOR), at the European level (CEN) and at the International Level (ISO) in the domain of Industrial Data and Advanced Manufacturing Technologies. Her work is focused on the standardization of product and process management data and on the joint application of the emerging standards: ISO 10303 STEP, MANDATE and PSL.

\section{R.I.M. Young}

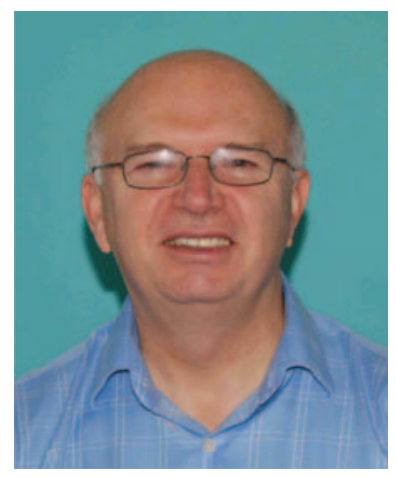

Dr Young is the programme director for Product Design and Manufacture at the Wolfson School of Mechanical and Manufacturing Engineering at Loughborough University in the UK. He has some 30 years experience in product design and manufacturing engineering, working both in UK industry and in academia. His research over the last 20 years has focused on decision support systems for manufacture with particular emphases on issues concerning design and manufacturing systems integration and interoperation. This has led to work in process planning, design for manufacture, product life cycle management, information modeling and knowledge sharing which has achieved funding from the UK government and industry of over È2M. Dr Young has published over 80 international research papers and serves on the scientific committee of numerous international conferences. $\mathrm{He}$ is an member of the UK 'Advanced Manufacturing Technology' committee and is also a UK expert to the International Standards Organization committee concerned with industrial automation and integration.

\section{Jean-Jacques Michel}

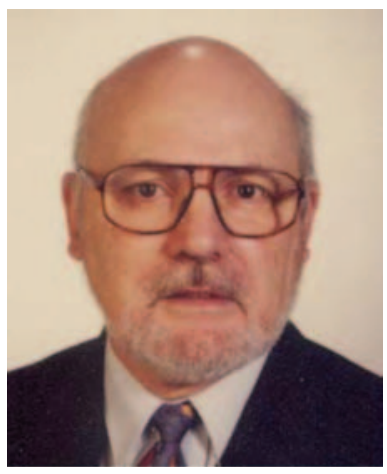

J.J. Michel was teacher and researcher in Atomic Physics and Spectronomy at the University Paris XIII (ORSAY) from 1963 to 1975 . He became then MIS Manager at the University Paris XIII, General Motors France and Pierre Fabre Laboratories (Pharmaceutical Industry) from 1975

to 1985. Then, he became MIS Manager at CETIM (French technical center for mechanical industry) in charge of standardization for Advanced Manufacturing Technologies (CIM) from 1985 to 2001. He has been TC184/SC5/WG1 expert since 1990, IEC SC65A_TC184/SC5/JWG15 Expert since its creation, TC184/SC4_SC5 JWG8 convener since 1996 and TC154/WG1 Expert from 2002 on.

$\mathrm{He}$ was Head of the French delegation to CEN/ TC310 (European Mirror committee of TC184) from its creation to 2002 and also French expert to CEN/STAR (CEN group in charge of the relations between European R\&D and European standardization) from its creation to 2002. He was also project leader of European Leonardo project FORMEDI. He is Chairman of the French mirror committee of TC $184 / \mathrm{SC} 5$. He is now Consultant in Standardization for French Companies (Schneider, Renault, etc...) 


\section{Reyes Grangel}

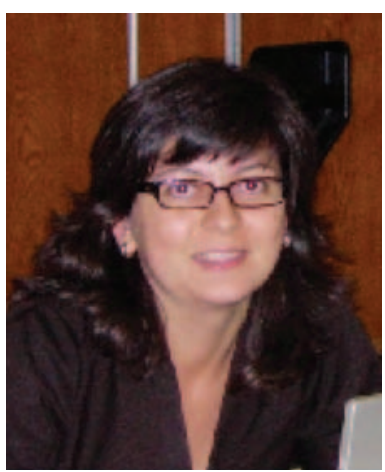

R. Grangel is assistant professor in Computer Science at the Department of Computer Languages and Systems at the Universitat Jaume I in Castelló (Spain). She is also member of the Research Group 'System Integration and ReEngineering' (IRIS Research Group). She received her BS in

Computer Engineering from the Universitat Jaume I in 1998. She has worked in several projects in the domain of systems integration, ERP implementation, and business process re-engineering in the tile industry and transport enterprises. Her research interests include reference architectures, enterprise modeling, modeldriven engineering and knowledge modeling, with the objective of integrating collaborative enterprises using enterprise modeling and business process re-engineering. She is now involved in the INTEROP NoE, especially in the TG2 'model driven interoperability'.

\section{Julie Le Cardinal}

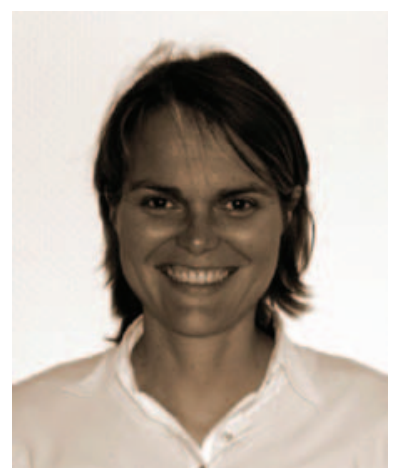

Julie Le Cardinal is assistant professor in the Industrial Engineering Laboratory at Ecole Centrale Paris, France. Her research focuses on the development of new methodologies of design and management in an industrial context. She graduated as mechanical engineer at the University of Technology of Compiègne, France, in the domain of industrial design, project methodologies (value analysis and project management (applications on concrete and varied industrial cases). She has got a Master of Industrial Engineering Systems, at the Ecole Centrale Paris, with a study about know-how capitalization of in design. The subject of her PhD Thesis is A Study of Dysfunctions within the Decision Making Process. Particular Focus on the Choice of Actor. The thesis deals with the optimization of the decision process in industrial projects: analysis of various industrial projects, validation of the proposed models within an industrial framework (Vallourec: meetings with project managers, to analyze dysfunctions in their projects and proposition of action plan and recommendations).

\section{Jean-Pierre Bourey}

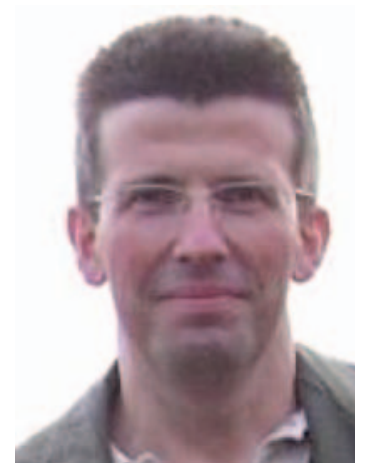

J.P. Bourey is professor in Software Engineering at the Ecole Centrale de Lille (France) where he has been responsible of both the last year program in Software Engineering and Information systems since 1989 and the Research Master Program in Information Systems and Design Engineering since 2003. He is in charge of the Laboratory of Industrial Engineering of Lille.

His teaching interests focus on software engineering, UML, object methodologies, information system, Ada language and model driven engineering. His recent research activities are related to his membership to the INTEROP network of excellence and more particularly to MDA-based transformation techniques to improve interoperability of enterprise software applications in the framework of TG2 'model driven interoperability'. 